\title{
El estallido social en Chile desde el igualitarismo relacional de Elizabeth Anderson
}

\author{
[The Chilean social uprising according to the relational \\ egalitarianism of Elizabeth Anderson] \\ Daniel Brieba (1) \\ Universidad Adolfo Ibáñez
}

\begin{abstract}
Resumen
En este ensayo se propone una reflexión sobre el estallido social chileno de 2019 a partir de la visión normativa de igualitarismo relacional de Elizabeth Anderson. Si la base de la protesta social fue una rebelión frente a situaciones y relaciones de abuso, desigualdad e indignidad, el igualitarismo relacional sería una perspectiva teórica que permitiría articular y responder a esas demandas. En este trabajo se argumenta que el ideal de igualdad democrática, entendido como el opuesto a una sociedad basada en relaciones jerárquicas, sugiere una hoja de ruta promisoria para el estallido y que trasciende las usuales disputas ideológicas chilenas en torno al rol del Estado y el mercado.
\end{abstract}

Palabras clave: protestas sociales, igualdad democrática, igualdad relacional, igualdad social, ideologías políticas.

\begin{abstract}
In this article, I reflect on the social uprising that occurred in Chile in 2019, according to the normative vision of relational egalitarianism of Elizabeth Anderson. I argue that, if the protests constituted a revolt against relationships and realities of abuse, inequality and indignity, then relational egalitarianism offers a substantial theoretical toolkit with which to articulate and respond to such complaints. In this article, I argue that the ideal of democratic equality constitutes a promising means of responding to the normative demands implicit in the October uprising, which transcend traditional Chilean ideological disputes concerning the respective roles of the State and the market.
\end{abstract}

Keywords: social protests, democratic equality, relational equality, social equality, political ideologies. 


\section{INTRODUCCIÓN}

El estallido social chileno de octubre de 2019 mostró que existe un malestar profundo y extendido en la sociedad chilena. El estallido no tuvo ni un petitorio ni vocerías, por lo que solo se puede interpretar inductivamente cuáles fueron sus demandas principales. Reconociendo que las raíces de este malestar no son unívocas (Peña, 2020), este ensayo plantea que al menos parte de ese malestar obedece a una sensación difundida en la ciudadanía de ser víctimas de abusos e injusticias institucionalmente ancladas en el orden económico y social imperante. Si esta rabia y malestar tienen causas económico-sociales abordables políticamente, cabe entonces preguntarse por el contenido de las demandas ciudadanas y las formas en que se podría responder a ellas.

Este ensayo parte de la hipótesis de que las tres demandas sustantivas centrales del estallido son aquellas por la dignidad, en contra del abuso y a favor de la igualdad. La demanda por dignidad fue omnipresente en las protestas. Ésta alude a la idea de que cada persona tiene una valía moral intrínseca que demanda respeto (Dillon, 2018), el cual tiene una dimensión material y una relacional (Brieba \& Bellolio, 2020). La primera se refiere a condiciones de vida materiales para vivir dignamente, es decir, a un piso mínimo o estándar de suficiencia, bajo el cual se estaría viviendo en condiciones indignas como ser humano o como ciudadano. Aquí la protesta se refiere a necesidades económicas objetivas y al reclamo por mejores servicios sociales ofrecidos por el Estado. La segunda dimensión se refiere a ser tratado por otros como un sujeto con dignidad y no como un medio para fines ajenos. El trato denigratorio y/o abusivo niega la dignidad que se realiza con un trato igualitario por otros. En Chile, la evidencia de que ambos tipos de dignidad son reclamos sentidos de la población es relativamente abundante (Araujo, 2016; Brieba \& Bellolio, 2020; PNUD, 2017). El abuso fue otro concepto usado de manera frecuente por los manifestantes del estallido. La sensación de abuso proviene de la percepción de que las élites económicas y políticas usan su poder para su propio beneficio, coludiéndose entre sí para protegerse mutuamente y beneficiarse a costa del resto. La percepción ciudadana mayoritaria es que la impunidad reina y que las reglas del juego están sistemáticamente torcidas a favor de los poderosos (Matamala, 2018).

A la base de este malestar también hay una demanda por mayor igualdad, que tiene varias caras. El reclamo por la igualdad de género se manifestó de manera más evidente en años y movilizaciones previas, pero en términos socioeconómicos hay también un reclamo contra una élite que es vista como demasiado cerrada, clasista, hereditaria, no meritocrática y que cuenta con una desmedida influencia sobre la vida política y económica del país (Matamala, 2018; Velasco \& Brieba, 2019). Si bien estos ámbitos tuvieron una elaboración menos explícita en las protestas sociales, las bicicletadas a casas de autoridades y las irrupciones en balnearios exclusivos y centros 
comerciales de sectores acomodados de Santiago dan una pista respecto al carácter anti-elitista de la protesta. En suma, dignidad, abuso y desigualdad son tres conceptos clave que resumirían el contenido político sustantivo del malestar de la sociedad chilena. ¿Tiene algo que decir la filosofía política al respecto? ¿Puede señalar posibles caminos coherentes ideológicamente para pensar estos problemas y proveer parámetros normativos de acuerdo a los cuales pensar soluciones y evaluar las medidas que se vayan proponiendo?

En lo que sigue intentaré mostrar cómo las ideas de Anderson (1999) son atingentes para mirar estos problemas y contribuir a la discusión política chilena. Propongo que las demandas centrales del movimiento social (i.e., dignidad, igualdad y fin a los abusos) tienen un correlato natural en el ideal de igualdad democrática que ha desarrollado Anderson en diversos trabajos (e.g., 1999, 2004, 2007a, 2007b, 2010, 2015, 2019). Sostengo que el igualitarismo relacional Andersoniano podría guiar un programa de cambios profundos y transformadores de la sociedad chilena, más allá de las disputas ideológicas sobre la (des)mercantilización de los derechos sociales que, a la luz de esta agenda, constituye un asunto relativamente secundario.

\section{IGUALITARISMO RELACIONAL: LA FILOSOFÍA POLÍTICA DE ELIZABETH ANDERSON}

La igualdad democrática

El punto de partida de la filosofía política de Anderson es su concepción de igualdad, que usualmente se concibe como un problema distributivo: cuánto de un bien relevante (e.g., ingresos, oportunidades) debiera dársele a cada cual, y cuánta desigualdad en dicho bien (si es que alguna) es permisible desde el punto de vista de la justicia. Sin embargo, Anderson (1999) postula que en las sociedades democráticas la igualdad crucial es relacional. Lo moralmente decisivo es que los ciudadanos estén en condiciones de relacionarse como iguales entre sí y pone su foco en los términos o condiciones bajo los cuales se desarrollan estas interacciones, y no en la comparación de cuánto de algún bien disponen las partes (Anderson, 2007b). Por ello, las distribuciones de ingresos y oportunidades tienen una importancia solo derivativa o instrumental, en tanto ciertas igualdades distributivas pueden ser necesarias para permitir, expresar o sostener relaciones sociales igualitarias. $\mathrm{Su}$ opuesto son las relaciones sociales jerárquicas, que se dan cuando cierto grupo cuenta con mayor estima, poder o estatus que otros, pudiendo así denigrar, dominar o ignorar a otros (Anderson, 2007b).

Para Anderson (1999), la igualdad democrática es el ideal de igualdad que debiera primar en sociedades democráticas y se constituye por un ideal negativo y uno positivo. Negativamente, la igualdad democrática implica la ausencia de relaciones sociales opresivas que ocurren cuando 
"alguna gente domina, explota, marginaliza, denigra e inflige violencia a otros (...) [ya que] identidades socialmente adscritas, roles distintivos en la división del trabajo, o diferencias en rasgos personales (...) jamás justifican relaciones sociales desiguales" (Anderson, 1999, p. 313). En un sentido positivo, la igualdad democrática busca constituir una comunidad en la cual sea posible la autodeterminación colectiva por medio del diálogo entre iguales bajo reglas aceptables a todos, en contraste con las relaciones jerárquicas e intrínsecamente desiguales defendidas por ideologías como el sexismo, el racismo, el clasismo, o las castas (Anderson, 1999).

Las dos condiciones centrales de la igualdad democrática son que se eviten relaciones privadas de opresión y que cada persona sea reconocida como un ciudadano igual en el espacio público. Para Anderson (1999), al igual que para el movimiento de los derechos civiles estadounidense, el espacio público incluye los espacios laborales, pues ser discriminado en ellos implica una posible relación privada opresiva y una lesión a la igual ciudadanía. Por ende, ambos requisitos son distintos, pero complementarios. Como ejemplifica Anderson (1999), una mujer que es sometida a una clitoridectomía está sometida a una relación privada opresiva, pero eso no lesiona necesariamente su igual ciudadanía en el espacio público y una ejecutiva que no es ascendida en virtud de su género no estaría siendo necesariamente oprimida, pero el techo de cristal lesiona su estatus de igual ciudadana.
Por la dignidad y contra el abuso

Tanto la dimensión material como la relacional de la demanda por dignidad en Chile se pueden entender desde el ideal de igualdad democrática. Partiendo por la material, para Anderson (1999) una persona en estado de pobreza o vulnerabilidad está expuesta a relaciones sociales opresivas pues, por ejemplo, quien no tiene más opciones de subsistencia podría terminar aceptando un trabajo peligroso, denigrante o sometido a relaciones abusivas por falta de alternativas. Para evitar esto, es fundamental asegurarle un piso robusto de acceso a condiciones materiales y no materiales que le entreguen la independencia necesaria. Este piso (e.g., de ingresos, educación, salud, pensiones, etc.) no ve los derechos sociales como mínimos que sirvan como protección contra la pobreza basada en la focalización de servicios sociales, como Atria (2014) ha caracterizado los mínimos del modelo chileno actual. Son, en cambio, pisos de ciudadanía, cuyo fin es garantizar la independencia de las personas para relacionarse con otros en pie de igualdad. Este no es un piso de mera subsistencia biológica, como el que propone Hayek (1944), sino propiamente un piso de igual ciudadanía, es decir, relacional, que va subiendo a medida que la sociedad se hace más próspera. El piso de suficiencia exigido por Anderson cubre todos los funcionamientos (Sen, 1999) que le permiten a cada cual funcionar como persona biológica y psíquica, como miembro productivo de la comunidad, y como ciudadano/a (cf, Anderson, 1999, pp. 317-318). De lograrse este piso, la 
dimensión material de la demanda por dignidad quedaría ampliamente cubierta.

Respecto a la dimensión relacional de la dignidad, la igualdad democrática es una teoría de justicia que, debido a que se enfoca en la igualdad relacional antes que la distributiva, le da un lugar central a las desigualdades de reconocimiento que siempre han perjudicado a los miembros de grupos subordinados. Los movimientos políticos igualitarios han buscado reconfigurar las relaciones sociales opresivas y generar las condiciones para poder funcionar como ciudadanos iguales (Anderson, 1999). Por ejemplo, gays y lesbianas exigen no ser discriminados u hostigados en espacios públicos, buscan cambios en las normas de sexualidad y género, $\mathrm{y}$ en las relaciones sociales de desprecio y hostilidad que los oprimen. Asimismo, personas en sillas de rueda no piden subsidios, sino infraestructura pública que les permita desplazarse por la ciudad y espacios de trabajo adaptados a sus necesidades, pues "ninguna redistribución de recursos divisibles puede asegurar las libertades que estos grupos buscan" (Anderson, 1999, p. 313).

Por ello, desde el punto de vista de la igualdad democrática las demandas feministas que irrumpieron en el estallido social son un reclamo contra la desigual ciudadanía del mismo modo en que lo son las demandas contra el abuso de las elites. En ambos casos, lo que subyace es una percepción de desigualdad relacional, anclada en factores estructurales (i.e., culturales, sociales y económicos), que le permite a ciertos grupos (e.g., hombres o la elite) mantener jerarquías de poder $\mathrm{y}$ estatus sobre las cuales se fundan privilegios no justificables interpersonalmente frente al resto. Puesto que la igualdad democrática requiere que cada persona pueda aparecer y funcionar como un pleno igual en el espacio público, cualquier norma o práctica que preserve o introduzca jerarquías de poder, estima o estatus entre personas o grupos es objetable. Por ello, las ideologías que justifican y naturalizan jerarquías y trato desigual entre grupos (e.g., clasismo, sexismo) no tienen cabida legítima en una sociedad democrática. Debido a este fuerte requerimiento igualitario la igualdad democrática es un ideal que busca combatir las causas de los tratos denigrantes o abusivos y limitar los diferenciales de poder que permiten a ciertos grupos torcer las reglas a su favor o lograr grados de impunidad por su mala conducta.

Por la igualdad

El ideal de la igualdad democrática no se agota en los pisos de suficiencia y las normas relacionales antes descritas. En varios trabajos, Anderson (2007a, 2007b, 2015, 2019) ha desarrollado diversas propuestas que se pueden agrupar en dos mecanismos adicionales para realizar el ideal de redistribución económica y el control de las elites.

Sobre la redistribución económica, Anderson (2007b) postula que, en lugar de idear mecanismos alternativos al mercado para asignar recompensas (que le parece impracticable y perjudicial), lo que debe hacerse es constreñir el rango de desigualdades aceptables a través del sistema de impuestos y transferencias. Eso supone el piso de mínimos antes desarrollado, pero también 
restricciones en el centro y el polo superior de la distribución de ingresos. En el centro, reclama por esquemas de seguridad social de corte universalista que protejan los ingresos y seguridad económica no solo de los más pobres, sino también de la clase media. Esto porque los programas universales evitan estigmatizar a los más pobres y, además, porque para una sociedad de iguales es deseable que la mayoría de la gente esté concentrada al centro de la distribución en lugar de tener una distribución bimodal de ingreso, lo cual podría ocurrir cuando las ventajas y desventajas que produce el mercado son acumulativas. En el polo sur, la acumulación de riqueza extraordinaria es problemática pues ésta puede usarse para convertirla en jerarquía, en la medida en que el ingreso se pueda usar para comprar diversas formas de poder, influencia y estatus. Sin limitaciones a la riqueza, la preocupación es que los ricos pueden capturar la agenda política y las políticas públicas que protejan sus intereses, transformando un proceso democrático en uno plutocrático y posibilitando la aparición de dinastías familiares, fenómeno que ha ocurrido en América Latina.

Respecto al control de las élites, (Anderson, 2007a) señala que las élites funcionales están compuestas por aquellos que ocupan los puestos altos en las jerarquías productivas y políticas, y profesionales de alto nivel. A diferencia de las élites sociales basadas en el apellido u otras características adscritas, que no cumplen una función social que legitime su existencia, las élites funcionales son necesarias en un esquema eficiente y productivo de división del trabajo. Por ello, más que abolir las élites funcionales, lo que se requiere es domesticarlas. Esto se puede hacer de dos maneras: diversificando su composición y limitando sus atribuciones. La diversificación de la composición de la élite se lograría igualando oportunidades de acceso y mediante mecanismos explícitos de selección que potencien la diversidad.

Respecto a la igualdad de oportunidades, una sociedad democrática debe evitar una situación donde los pobres reciban una peor educación que los ricos generando así escasa movilidad dentro y entre generaciones. Ello relegaría a los pobres a una ciudadanía de segunda clase, con acceso solo a cierto tipo de trabajos. En cambio, debe haber posibilidades justas de éxito para todos en la competencia por los buenos trabajos (Anderson, 2004; Satz, 2007). Ello requiere una distribución de oportunidades educativas tal que todos los niños y niñas, independientemente de su origen social, tengan acceso a una educación escolar de suficiente calidad como para permitirles acceder a una carrera universitaria seria, provistas de condiciones de esfuerzo académico que sean sustanciales, mas no extraordinarias (Anderson, 2007a). Si bien este estándar no requiere una igualdad perfecta de oportunidades (e.g., no hay un requerimiento de que haya igual oportunidad de entrar a la carrera más prestigiosa de la universidad más prestigiosa), es claro que un estándar como éste no ha sido alcanzado aún por el sistema educativo chileno y probablemente por ningún sistema latinoamericano.

Respecto a los mecanismos de selección, Anderson (2007a) indica que los reclutadores de élites, como las universidades que las forman y, presumiblemente, las empresas, debieran seleccionar a sus integrantes explícitamente en 
aras de generar una elite socialmente diversa, que incluya una cantidad significativa de miembros de todos los grupos relevantes de la sociedad. El argumento para justificar esto redefine lo que constituye una selección meritocrática para puestos de élite: más que pensar en dichos puestos como premios a repartir entre individuos especialmente merecedores, esos puestos solo existirían y se justificarían en función del bien que proveen a la sociedad en su conjunto. Esto implica que las elites deben constituirse "para ser sistemáticamente responsivas a los intereses y preocupaciones de gente de todas partes (...). Esta concepción democrática de las responsabilidades de una élite debiera moldear nuestra concepción de las calificaciones que ésta debe tener" (Anderson, 2007a, p. 596). Desde esta perspectiva, las credenciales académicas son solo una parte de las calificaciones que una élite debe poseer. De manera más fundamental, una elite socialmente heterogénea servirá mejor los intereses de los diversos grupos que componen una sociedad porque conocerá mejor sus problemas, tendrá mayor disposición a atenderlos, y podrá comunicarse de manera más eficaz con dichos grupos, siendo así una elite más competente. En cambio, una elite socialmente homogénea (e.g., sacada solo del sector acomodado de Santiago, de un conjunto limitado de colegios y carreras universitarias, abrumadoramente masculina y de mayores de 50 años) sufrirá severos déficits cognitivos respecto a los problemas de otros grupos sociales y/o carecerá de la suficiente motivación para atenderlos. Es plausible pensar que en la génesis del estallido social estuvo precisamente la percepción de ser gobernados por una élite radicalmente ignorante de-e indiferente frente a-los problemas de la gran mayoría de los chilenos.

La segunda forma en que se pueden domesticar las élites funcionales es limitando sus atribuciones. Esto se refiere a la limitación de la autoridad de jefes y superiores jerárquicos en el trabajo. Para Anderson (2015, 2019), uno de los grandes errores del pensamiento político y económico moderno es imaginar el mundo del trabajo como un gran espacio de libertad negativa, donde impera la libertad de cada cual para transar su fuerza de trabajo a cambio de una remuneración. Esta caracterización olvida que, una vez que el trabajador se ha vuelto un empleado de una empresa, el trabajador está sujeto a sus reglas y relaciones de autoridad $\mathrm{y}$, potencialmente, a relaciones de dominación. Así, desde el punto de vista de la libertad republicana (i.e., no estar sujeto a la voluntad arbitraria de ningún otro) las relaciones laborales en una organización no son un espacio de libertad, sino su falta. La subordinación y dependencia, no la libertad, son el sello del trabajo asalariado moderno. Anderson (2015) llama a la forma de gobernanza dentro de la empresa gobierno privado, es decir, uno que no rinde cuentas a los gobernados y que tiene poder arbitrario sobre ellos.

Cuando los subordinados están sujetos a la voluntad arbitraria de los jefes -y esa sería la condición normal para la gran mayoría de los trabajadores, especialmente aquellos al fondo de la jerarquía organizacional-el riesgo de abuso de dicha autoridad es evidente. El problema no es que los jefes den órdenes sobre el trabajo y el 
cómo hacerlo, sino que los jefes pueden usar su autoridad de forma abusiva, a menos que haya leyes que específicamente lo constriñan, prohibiendo, por ejemplo, a sus empleados ir al baño, sometiéndolos a inspecciones corporales denigrantes, a violencia física y psicológica, o a condiciones de trabajo peligrosas. También pueden usarla para fines ajenos a la empresa, como obligar a sus empleados a contribuir a cierta causa o sancionarlos por su conducta $u$ opiniones fuera de ésta. Los problemas de abuso laboral no son ajenos a la realidad chilena, donde además interactúan con las dinámicas verticales y clasistas propias de la realidad latinoamericana (Rodríguez, 2010). Por todo esto, Anderson (2019) sugiere que se debiera repensar de manera bastante fundamental los términos de la gobernanza dentro de la empresa para sujetarla a un Estado de Derecho interno que le quite arbitrariedad al gobierno empresarial y les dé mayor voz y participación a sus trabajadores. Así, se busca que ser empleado de una empresa solo implique estar sujeto a una autoridad estrictamente circunscrita y no arbitraria, como ya ocurre respecto a la autoridad pública (Anderson, 2019).

Igualitarismo relacional y política chilena

Las ideas revisadas aquí no son, en su gran mayoría, parte de la discusión chilena actual que ha sido muy poco influenciada por la tradición liberal igualitaria anglosajona, de la cual Anderson es parte. Por ello, es legítimo pensar cómo se relacionarían sus ideas con aquellas dominantes en la izquierda y derecha chilenas.
Para la derecha chilena (o al menos para su parte dominante en los últimos 30 años, que podría denominarse Chicago-gremialista) la idea de libertad negativa o de no-interferencia es una piedra angular de su filosofía política (Velasco \& Brieba, 2019). Por ello, le produce cierto conflicto una filosofía que parte desde la idea republicana de libertad: la no dominación. Este disímil punto de partida redunda en que políticas como la seguridad social universal, la limitación de la autoridad legal de las empresas sobre sus trabajadores, y las exigencias de integración social en escuelas, universidades y reclutamiento de las élites, sean políticas que generen incomodidad en tanto interfieren con dicha libertad negativa. Por otra parte, el combate frontal a las normas y prácticas que alientan dinámicas clasistas o sexistas (o que defiendan jerarquías grupales en general) puede generar resistencia de facto en los sectores más conservadores o tradicionalistas de la sociedad chilena. Como Anderson (2010) además ha desarrollado un fuerte argumento en contra de la segregación (ya sea racial o por clases), y a favor de la integración en diversos ámbitos de la vida social, es fácil imaginar resistencias en sectores importantes de la élite chilena a requerimientos de este tipo.

Algunos aspectos de la igualdad democrática también le pueden generar incomodidad en la izquierda. En primer lugar, el reclamo que probablemente ha sido central de la izquierda chilena en la última década, como es la desmercantilización de los derechos sociales, está ausente en la agenda de Anderson. Esto se debe a que el foco de la autora está puesto en los 
resultados (e.g., acceso a pisos materiales de ciudadanía, igualación de oportunidades educativas) y no en el modo de provisión de éstos. $\mathrm{Si}$ dichos objetivos se logran con formas puramente públicas de provisión, o con una mezcla de proveedores, regímenes. y reglas, es una cuestión principalmente empírica: las preguntas de diseño institucional están al servicio de estos objetivos finales. En ese sentido, por ejemplo, Anderson no afirma que haya que prohibir los colegios particulares pagados, en la medida en que estos efectivamente entreguen una mejor educación que redunde en un beneficio colectivo gracias a contar con gente más o mejor calificada. Distinto sería si el principal beneficio que entregan es una red de contactos de exalumnos que les permita acceder por vías no meritocráticas a mejores puestos de trabajo, pues ahí no hay valor social agregado alguno sino un perjuicio directo a personas mejor calificadas (Anderson, 2004).

Este último punto le lleva a su argumento más general a favor de los mercados, el cual puede generar resistencia en sectores de izquierda, y en el cual coincide, singularmente, con Hayek (1944): que, con sus señales de precios, los mercados son un instrumento a favor de la productividad y la eficiencia. Esto es fundamental, ya que, desde un punto de vista normativo, colectivamente "compartimos un interés común abrumador a favor de la eficiencia" (Anderson, 2007b, p.252). Por ello, las políticas igualitaristas deben, en lo posible, acomodar esta realidad en vez de tratar de inventar mecanismos que nieguen el rol de los precios y los incentivos. La manera de combatir las consecuencias distributivas negativas de los mercados pasa por los pisos, techos y seguros universales discutidos anteriormente. La viabilidad de esta solución basada en impuestos depende de que la señal de precios pueda comprimirse sin deteriorar demasiado su calidad (Anderson, 2007b). Esta apuesta parece plausible al observar el éxito de los países del norte de Europa en este sentido.

Este último punto podría sugerir que el igualitarismo relacional no es sino otro nombre para la socialdemocracia en su versión europeacontinental y/o en una versión más cercana a la tercera vía que ejemplificaron gobiernos como los de Clinton en Estados Unidos, Blair en el Reino Unido, o los de la Concertación en Chile. Si bien en la idea de Anderson de constreñir las desigualdades económicas mediante el sistema impositivo hay una continuidad con la socialdemocracia, en otros aspectos hay diferencias importantes. En primer lugar, la socialdemocracia tiene un foco distributivo, no relacional. Su énfasis ha estado en limitar o reducir las desigualdades económicas, de resultados, o de oportunidades (Giddens, 2000). Por ello, la socialdemocracia es compatible con fuertes desigualdades de poder y con la presencia de relaciones opresivas no-económicas que perjudican a determinados grupos (e.g., mujeres, pueblos indígenas, minorías sexuales). En cambio, para el igualitarismo relacional toda forma de opresión y de desigualdad ciudadana es intrínsecamente injusta, sean sus causas socioeconómicas o no. En segundo lugar, incluso si nos limitamos al plano puramente socioeconómico, el objetivo de la socialdemocracia es reducir las 
desigualdades, pero sin especificar cuánta reducción es necesaria o aceptable. En Chile, la tercera vía concertacionista fue más acotada en sus ambiciones al solo buscar mejorar las condiciones de los que estaban peor. Sus triunfos en este sentido fueron importantes (e.g. el programa AUGE de salud, las pensiones mínimas, la disminución de la pobreza), pero desde un punto de vista filosófico, esta posición no califica como igualitarista sino como "prioritarista" (Parfit, 1997), pues consistió fundamentalmente en priorizar la mejoría en el bienestar de los más pobres antes que en garantizar o explicitar un piso de suficiencia material que posibilitara relaciones sociales auténticamente igualitarias (Velasco \& Brieba, 2019). Finalmente, una tercera diferencia radica en el fuerte acento que pone Anderson en la domesticación de las elites funcionales por medio de la activa diversificación de su composición y la limitación de sus atribuciones de mando organizacional. Esta visión democrática- republicana de las elites como un grupo que debe estar al servicio de los intereses de la sociedad es ajena a la tradición socialdemócrata, que a lo más buscó es limitar la transmisión intergeneracional del privilegio desde el estrecho marco conceptual de la igualdad de oportunidades. Si parte del descontento democrático que alimenta al populismo en el mundo es precisamente la percepción de que las élites han perdido contacto con-y el interés por-las necesidades y opiniones de la masa de ciudadanos (e.g. Collier, 2018; Fukuyama, 2018), esta diferencia es particularmente importante.

En suma, por al menos estas tres razones, el programa igualitario andersoniano es sustancialmente más comprehensivo y radical que lo que ha sido la socialdemocracia en sus diferentes versiones. También es más radical que la agenda de desmercantilización de derechos sociales, que de ser llevada plenamente a cabo, podría tener modestos efectos sobre las desigualdades de base y, en cualquier caso, cubre solo un aspecto del rango de las desigualdades objetables.

\section{CONCLUSIONES}

A pesar de la virulencia del estallido social chileno y de los meses que han transcurrido desde su inicio, lo más notorio del panorama político chileno actual es su ausencia de grandes ideas transformadoras. Esto puede parecer extraño en tiempos que se habla de manera permanente sobre cambiar el modelo y donde habrá un plebiscito para aprobar una nueva Constitución y una Asamblea Constituyente.
Con todo, a nivel de contenidos sustantivos, las grandes ideas escasean.

La tesis de este ensayo es que, si efectivamente las quejas de fondo que se manifestaron en el estallido social son las demandas por la dignidad, la igualdad y el fin de los abusos, el ideal de igualdad democrática de Anderson propone un programa sistemático, radical e internamente coherente de cambios sociales para avanzar en 
esa dirección. En ese sentido, esta agenda es considerablemente más ambiciosa y comprehensiva que las que actualmente se discuten en Chile por los principales sectores políticos.

A pesar de las resistencias que estas ideas puedan generar tanto en la izquierda como en la derecha, no es menos cierto que amplios grupos en ambos sectores podrían abrazar una agenda que no es ni anti-Estado ni anti-mercado. La igualdad democrática plantea un camino que permitiría mantener un sistema económico productivo con libertad de precios y realizar un exigente ideal de igualdad social, temáticas que preocupan tanto a la derecha como a la izquierda.

\section{REFERENCIAS}

Anderson, E. (1999). What is the point of equality? Ethics, 109(2), 287-337. doi: 10.1086/233897

Anderson, E. (2004). Rethinking equality of opportunity: Comment on Adam Swift's how not to be a hypocrite. Theory and Research in Education, 2(2), 99-110. doi: 10.1177/1477878504043438

Anderson, E. (2007a). Fair opportunity in education: A democratic equality perspective. Ethics, 1174), 595-622. doi: 10.1086/518806 Anderson, E. (2007b). How should egalitarians cope with market risks? Theoretical Inquiries in Law, (1), 239-270. doi:10.2202/1565-3404.1175 Anderson, E. (2010). The imperative of integration. Princeton and Oxford, NJ/UK: Princeton University Press.

Anderson, E. (2015). Equality and freedom in the workplace: Recovering republican insights. Social Philosophy and Policy, 31(2), 48-69. doi:10.1017/S0265052514000259 Anderson, E. (2019). Private government: How employers rule our lives (and why we don't talk about it). Princeton and Oxford, NJ/UK: Princeton University Press.
Araujo, K. (2016). Desigualdades interaccionales e irritaciones relacionales: Sobre la contenciosa recomposición del lazo social en la sociedad chilena. (Documento de Trabajo $\mathrm{N}^{\circ} 3$ ). Recuperado del sitio de Internet del Centro de Estudios de Conflicto y Cohesión Social (COES): https://www.coes.cl/wp-content/uploads/N03.pdf Atria, F. (2014). Derechos sociales y educación. Santiago, Chile: LOM Ediciones.

Brieba, D., \& Bellolio, C. (2020). No country for liberals? El estallido social chileno: Una interpretación rawlsiana. En, B. Ugalde, F. Schwember \& V. Verbal (Eds.), El octubre chileno. Reflexiones sobre democracia $y$ libertad (pp. 249-272). Santiago, Chile: Ediciones Democracia y Libertad.

Collier, P. (2018). The future of capitalism. Facing the new anxieties. New York, NY: Harper Collins.

Dillon, R. S. (2018). Respect. En E. Zalta (Ed.), The Stanford encyclopedia of philosophy. Recuperado de https://plato.stanford.edu/archives/spr2018/entr ies/respect/. 
Brieba, D. (2020). El estallido social en Chile desde el igualitarismo relacional de Elizabeth Anderson. Revista de Sociología, 35(1), 31-42. doi: 10.5354/0719529X..2020.58105

Fukuyama, F. (2018). Identity. London, UK: Profile Books.

Giddens, A. (2000). The third way and its critics. Cambridge, UK: Polity Press.

Hayek, F. (1944). The road to serfdom. London, UK: Routledge.

Matamala, D. (2018). Los reyes desnudos. Santiago, Chile: Editorial Catalonia.

Parfit, D. (1997). Equality and priority. Ratio, 10(3), 202-221. doi: 10.1111/1467-9329.00041

Peña, C. (2020). Pensando el malestar. Santiago, Chile: Editorial Taurus.

Programa de las Naciones Unidas para el Desarrollo [PNUD] (2017). Desiguales. Orígenes, cambios y desafios de la brecha social en Chile. Santiago, Chile: Programa de las Naciones Unidas para el Desarrollo.
Rodríguez, J. K. (2010). Employment relations in Chile: Evidence of HRM practices. Articles Relations Industrielles, 65(3), 424-446. doi: 10.7202/044890ar

Satz, D. (2007). Equality, adequacy, and education for citizenship. Ethics, 1174), 623-648. doi: 10.1086/518805

Sen, A. (1999). Desarrollo y libertad. Bogotá, Colombia: Editorial Planeta.

Velasco, A., \& Brieba, D. (2019). Liberalismo en tiempos de cólera. Santiago, Chile: Editorial Debate.
Manuscrito recibido: 12-04-2020

Manuscrito aceptado: 27-06-2020 